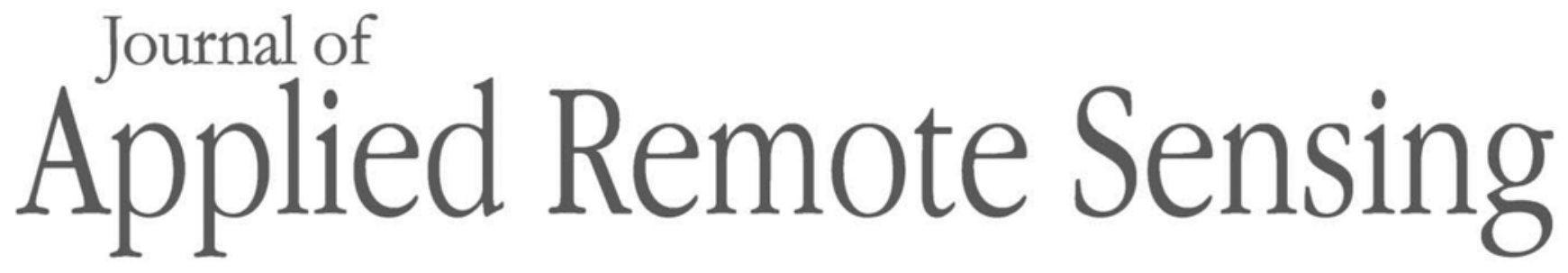

RemoteSensing.SPIEDigitalLibrary.org

\title{
Special Section Guest Editorial: Advances in Remote Sensing for Forest Structure and Functions
}

\author{
Lin Cao \\ Yunsheng Wang \\ Hao Tang
}




\title{
Special Section Guest Editorial: Advances in Remote Sensing for Forest Structure and Functions
}

\author{
Lin Cao, ${ }^{a}$ Yunsheng Wang, ${ }^{\mathrm{b}}$ and Hao Tang ${ }^{\mathrm{c}}$ \\ ${ }^{a}$ Nanjing Forestry University, Co-Innovation Center for Sustainable Forestry in Southern China, \\ Nanjing, China \\ ${ }^{b}$ Finnish Geospatial Research Institute FGI, National Land Survey, Masala, Finland \\ 'University of Maryland College Park, Department of Geographical Sciences, College Park, \\ Maryland, United States
}

Forest is one of the most important ecosystems, accounting for $75 \%$ of terrestrial gross primary production. The vertical structure of a forest plays a key role in its ecosystem process and function and provides crucial implications for forest resources management. Such importance raises a great interest in developing advanced remote sensing methodologies for automatically extracting forest structural information, with a focus on linking forest structural characteristics to multiple forest functions by using multiple sensors and platforms. Many processes were made using a great variety of remote sensing technologies, such as spaceborne, airborne, unmanned aerial vehicle (UAV), terrestrial mobile and stationary. Together, these platforms offer a synoptic view over broad geographical area and at unprecedented high accuracy, providing key data sources for monitoring the forest structure and filling gaps in current forestry research.

From the point of view of sensors, light detection and ranging (LiDAR) and photogrammetry are among the most popular techniques for measuring vertical forest structure. While LiDAR data is a state-of-the-art source for highly precise 3D measurements, structure from motion (SfM) techniques is capable to carry out 3D measurements in a similar way as the LiDAR point clouds. In particular, recent development of UAV platforms have enabled end-users to overcome both financial and technical difficulties in using aircraft and satellite data, which greatly stimulated the application of UAV-based LiDAR and imagery in forest communities to measure forest structures, and to further evaluate and/or predict forest functions. Another major advantage of UAV point cloud data, be it LiDAR or SfM sourced, is the much higher point density and spatial resolution that is capable to provide detailed structural information not available from airborne and satellite platforms.

This special section aims to provide a timely update on the latest development of remote sensing techniques for acquiring forest structure and function information. It contains 13 independent studies that cover several major aspects under the broad scope in remote sensing of forest structure: (1) individual tree segmentation, (2) tree species classification, (3) retrieval of forest biophysical variables, and (4) novel experimental design and algorithm development. Zhou et al. delineated individual tree crown using superpixel and topological features based on ultra-high spatial resolution UAV imagery. Feng et al. improved the accuracy of tree species classification through combined usage of spatial features derived from airborne hyperspectral imagery and LiDAR data. Denux et al. compared multiple algorithms to determine the best practice of time series analysis under a coarse spatial resolution. Xu et al. compared the capacity of LiDAR and UAV structure from motion (UAV-SFM) data in estimating the stand characteristic parameters of subtropical forests. Kanja et al. explored the benefit of combing structural, spectral and texture metrics in estimating forest parameters. $\mathrm{Ni}$ et al. proposed a data fusion approach for monitoring the forest tree heights and their changes by using GLAS and Landsat TM data. $\mathrm{Xu}$ et al. jointly used LiDAR and SAR data to retrieve forest canopy height through a random volume over ground (RVoG) model. Zhan et al. focused on the importance and sensitivity of forest productivity at the canopy level using continuous observations. Zhang et al. improved UAV-based hyperspectral imagery acquisition with a novel semiautomatic multi-angle method.

These studies indicate a new trend that shows UAV-based remote sensing is becoming a more popular art in estimating the forest structures and functions, such as aboveground biomass, tree

(C) 2020 Society of Photo-Optical Instrumentation Engineers (SPIE) 
volume, and carbon flux. Both LiDAR and SfM point clouds at times show similar performance in estimating forest parameters and their biomass. In addition, the multi-angle view capacity also provides much higher flexibility in experimental designs. However, the use of UAV alone cannot fully meet the needs of long-term monitoring tasks. To fully understand how forest structure and function changes under the changing climate, it is critical to establish a more comprehensive observation network including UAV, aircraft, and satellite data collections over long time spans.

Finally, we would like to thank the authors of the special section for their contributions. Great appreciations to the reviewers and the Journal of Applied Remote Sensing editorial team for conducting a high-quality review process for all of the published papers. We hope that this special section will foster further development of forest remote sensing sciences and contribute to formation of forest management plans and policies.

Lin Cao is a professor in the College of Forestry at Nanjing Forestry University (NJFU), China. He received a PhD from the University of British Columbia (UBC), Canada, in 2016. His research interests are LiDAR applications in forest inventory and management, UAV and point cloud processing, forest biomass estimation, and forest change detection.

Yunsheng Wang is a senior research scientist with the Centre of Excellence in Laser Scanning Research, Academy of Finland, Finland, and with the Finnish Geospatial Research Institute, Finland. She received PhD from the University of Freiburg, Germany, in 2008. Her main research interests lie in the assessment of ecosystem services and 3D modeling from point cloud data.

Hao Tang is an assistant research professor in the Department of Geographical Sciences at University of Maryland College Park, where he also received his PhD in 2015. He is currently working on NASA's Global Ecosystem Dynamics Investigation (GEDI) mission as a member of the science team. He is also a recipient of NASA Earth and Space Science fellow (NESSF) and NASA New (Early Career) Investigator Program. His primary interests focus on characterizing 3D dynamics of terrestrial ecosystems using different LiDAR remote sensing platforms. 